\title{
Improved 3D Reconstruction Method for Range-Gated Laser
}

\author{
Shuyu Wang ${ }^{a}$, Fan Yang ${ }^{b}$, Chunsheng $\mathrm{Hu}^{\mathrm{c}}$ and Chong Lin ${ }^{\mathrm{d}}$ \\ Army artillery Air Defense Academy of PLA, Anhui 230031, China \\ a1512264822@qq.com, b13865513296@126.com, c6533427@qq.com, dlingdirk2009@163.com
}

\begin{abstract}
Range-gated laser imaging obtains different range-gated images by imaging the target slice. However, range-gated laser imaging cannot provide the full spatial position information of longrange targets. Thus, this paper proposes an algorithm that combines the threshold and crosscorrelation methods for high-precision 3D reconstruction. First, the threshold value method is used to eliminate the zero point in the measured gray-scale curve. Then, the interceptive and ideal grayscale curves measured at each pixel point are cross-correlated, and the delay that corresponds to the correlation peak at each pixel point is used to calculate the range value at each pixel point. The $3 \mathrm{D}$ coordinates at each pixel point can be calculated based on the imaging system parameters. Finally, the long-range arbor slice image is processed to obtain the 3D arbor image.
\end{abstract}

Keywords: laser, range-gated, 3D reconstruction.

\section{Introduction}

Range-gated laser active imaging technology can overcome atmospheric backscattering to image long-range targets. A long-range intensity image and the range value corresponding to each slice of image are obtained. The target image collected in this system is only a 2D image, which seriously affects the recognition of information, such as the target contour and orientation. 3D images have attracted increasing attention because they can provide additional information and realistic images than $2 \mathrm{D}$ images. At present, relatively few findings were found about the $3 \mathrm{D}$ reconstruction of rangegated laser active imaging of long-range targets.

The $3 \mathrm{D}$ reconstruction method mainly involves surface, volume, and point rendering methods. The surface rendering method requires short distances between each slice[1]. Thus, the surface rendering method is unsuitable for $3 \mathrm{D}$ reconstruction due to the long ranges involved. Therefore, only the volume and point rendering methods are applied to the $3 \mathrm{D}$ reconstruction of range-gated slice images. Yang Jinhong et al. [2] used the volume rendering method to perform 3D reconstruction of an underwater target on the basis of range-gated imaging. Given the low signal-to-noise ratio of the laser image and the complexity of the volume rendering algorithm, the $3 \mathrm{D}$ reconstruction results could not intuitively represent the spatial location information of the target. Jens Busck et al. [3] used Lidar technology to calculate the range value at each pixel by using the gray-scale curve and the data weighted average method, thereby achieving high-precision $3 \mathrm{D}$ reconstruction through $3 \mathrm{D}$ coordinates at each pixel. The gray-scale curve at each pixel of a range-gated slice image is flattopped and seriously affected by noise. Therefore, this method is unsuitable for the 3D reconstruction of long-range target images collected through the range-gated system. Pierre Andersson et al. [4] used the data feature positioning method to calculate the pixel range value based on the range gating system and then calculate the 3D coordinates of the pixel. The range value obtained by this method is not highly accurate because the appropriate delay on the gray scale curve can be selected through the set threshold value.

The 3D reconstruction method described above is compared and analyzed in terms of the characteristics of the range-gated laser active imaging system [5-6]. The point rendering method is suitable for the $3 \mathrm{D}$ reconstruction of long-range targets through range-gated $2 \mathrm{D}$ images. The similarity of the measured gray-scale curve formed by different gray-scale values of the pixel points on each slice image is different from that of the ideal gray-scale curve. Thus, the effective range information can be obtained. The correlation function is widely used in the recognition of radar echo, in the communication synchronization of signals, and in other fields because this function is a powerful tool for discriminating signals. Therefore, the correlation function can be introduced to describe the similarity of the laser echo and ideal signals. The different delays that correspond to the 
peak value of the cross-correlation curve of the ideal and measured gray-scale curves at each pixel can be used to calculate the range value of each pixel. The high-precision $3 \mathrm{D}$ coordinates of the pixel point can be obtained together with the geometric parameters of the imaging system. Many zero points exist in the measured gray-scale curve, and these points affect the cross-correlation results, thereby reducing the accuracy of $3 \mathrm{D}$ reconstruction. Thus, this paper proposes an algorithm that uses the interceptive gray-scale curve obtained through the threshold method and the distance information extracted through the cross-correlation method to perform high-precision 3D reconstruction of longrange targets.

\section{Basic Principle}

\subsection{Ideal Gray-scale Curve}

The range-gated laser active imaging system can change the delay time according to the set step to obtain the gray-scale curve at each pixel. The gray-scale value of each pixel in the range-gated image is proportional to the integral of the product of the laser pulse and the detector shutter returned in the instantaneous field of view. For the general YAG laser, the laser pulse model [7] is:

$$
P(t)=(t / \tau)^{2} \exp (-t / \tau)
$$

where "P" is the laser pulse power, and " $\tau$ " is the laser pulse width.

For simplification, the receiving detector ICCD high-speed shutter, " $g(t)$ ", is assumed to be a rectangular pulse under the ideal condition depending on the delay time. Without considering the noise, the normalized pixel gray-scale curve is the integral of the product of the normalized laser pulse and the receiving detector shutter, that is[8],

$$
G\left(t_{0}\right)=\int_{0}^{\infty} P(t) g(t) d t=\int_{t_{d}}^{t_{d}+\tau_{g}} P(t) d t
$$

where "G" is the ideal gray-scale curve, "P" is the returned laser pulse power, " $\mathrm{g}$ " is the receiving detector shutter, " $\mathrm{td}$ " is the delay time, and " $\mathrm{g}$ " is the time of the receiving detector ICCD shutter.

The pulse width of the returned laser pulse in the experiment is $10 \mathrm{~ns}$ if the receiving detector shutter is $100 \mathrm{~ns}$. Fig. 1 shows the normalized ideal gray-scale curve obtained by simulating the integral of the ideal return laser pulse and the receiving detector shutter[9].

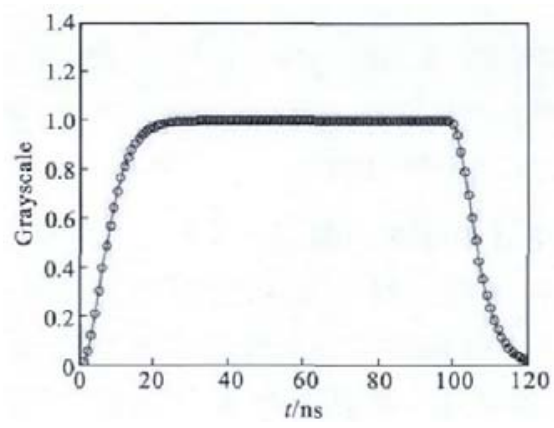

Fig.1 Ideal gray-scale curve

\subsection{Interceptive Gray-scale Curve Obtained Through Threshold Method}

In the experiment, 14 images are used for 3D reconstruction. The corresponding gray-scale value of each pixel in the 14 images is calculated and divided by the maximum gray-scale value. Then, the normalized gray-scale curve measured at each pixel point is obtained by interpolating 14 gray-scale values. The measured gray-scale curves of the randomly selected points $(288,302)$ are shown in Fig. 2 . 


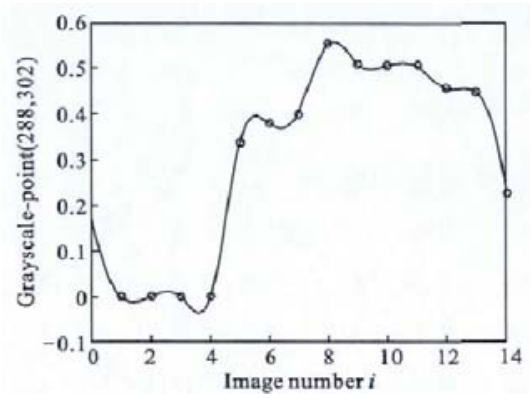

Fig. 2 Normalized gray-scale curve of point $(288,302)$

The front-end zero value of the measured gray-scale curve does not provide effective range information and thus affects the cross-correlation between the measured curve and the ideal curve and greatly reduces the accuracy of the subsequent $3 \mathrm{D}$ reconstruction. Thus, the threshold value can be set to reject the point with the least gray-scale value, that is, the point with less information. Fig. 3 shows the measured gray-scale curve after the zero point is rejected randomly $(288,302)$.

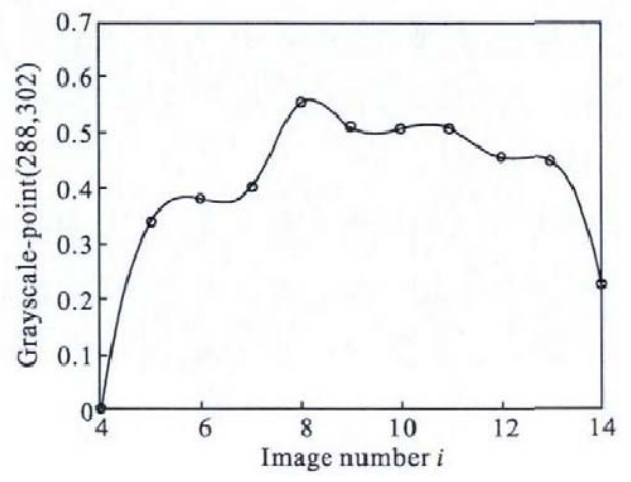

Fig. 3 Measured normalized gray-scale curve of point (288,302) after rejecting zero points

\subsection{Calculate the Pixel Distance Through the Cross-Correlation Method}

Given that the gray-scale curve exists in the form of a point sequence in MATLAB, the correlation function of discrete signal should be used when calculating the similarity between the measured and ideal gray-scale curves.

The cross-correlation function of signals " $x(n)$ " and " $y(n)$ " is defined as:

$$
r_{x y}(m)=\sum_{n=-\infty}^{\infty} x(n) y(n+m)
$$

where "rxy(m)" at time " $m$ " is equivalent to the product of the two sequences when " $x(n)$ " remains unchanged and "y(n)" shifts to the left to the mth sampling period.

Samples of the measured and ideal gray-scale curves are taken at any interval to calculate the cross-correlation of the discrete signals. The sampling interval is set to $0.05 \mathrm{~ns}$, and the ideal and measured interceptive gray-scale curves of point $(288,302)$ are correlated to obtain the result shown in Fig. 4.

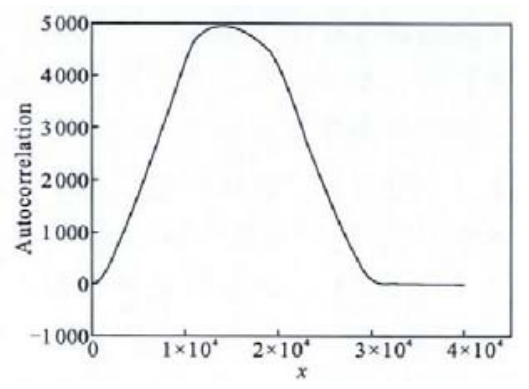

Fig. 4 Cross-correlation of the ideal and measured gray-scale curves of point $(288,302)$ 
Fig. 4 shows that a peak exists in the cross-correlation curve. Thus, the pixel range value can be estimated through the horizontal ordinate that corresponds to the peak value of the cross-correlation curve at different pixel points. The distance value of each pixel can be calculated by substituting the horizontal ordinate corresponding to the peak point in the cross-correlation curve of each pixel into Equation (4).

$$
L=\frac{c \times\left(t_{0}+\left(t_{1}+(x \times \Delta t) / 2\right) \times 10^{-9}\right)}{2}
$$

where " $\mathrm{L}$ " is the pixel range, "c" is the speed of light, "to" is the initial delay time, "tl" is the delay time corresponding to the first non-zero value point of the gray-scale curve, " $\triangle \mathrm{t}$ " is the point-to-point interval of the ideal and measured gray-scale curve point sequence, and " $x$ " represents the horizontal ordinate corresponding to the peak of the cross-correlation curve.

\section{Composition of Experiment System}

The experiment system is mainly composed of a laser emission system, an echo image acquisition system, and a central control processing system. The system is shown in Fig. 5. A YAG laser with a $532 \mathrm{~nm}$-long wavelength is used as the illumination source, a super-second-generation ICCD is used as the imaging device, and a minimum gate width is designed for the synchronous control circuit based on FPGA, with a minimum stepping time of $10 \mathrm{~ns}$.

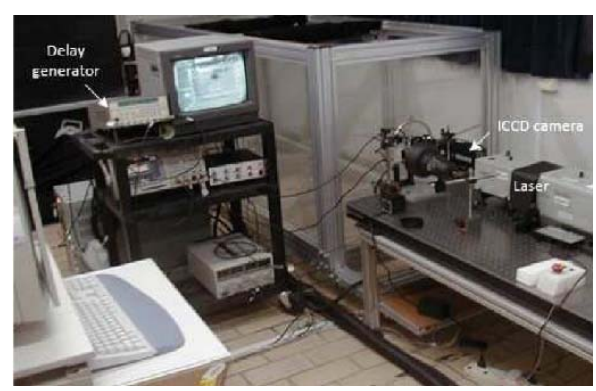

Fig. 5 Active laser imaging experiment system

Active laser imaging experiments are performed for the arbor with a range of approximately 1.2 $\mathrm{km}$. Sixteen sets of slice images at different ranges are obtained with an initial delay time of $10 \mathrm{~ns}$ delay stepping, a laser pulse width of $10 \mathrm{~ns}$, and an ICCD shutter of $100 \mathrm{~ns}$. Each group of images has 50 frames; the even array imaging results are shown in Fig. 6. The target is oblique in the image and cannot provide effective distance information. Therefore, the first and last frames are discarded during the $3 \mathrm{D}$ reconstruction, and the middle 14 images are used to perform the $3 \mathrm{D}$ reconstruction for the arbor.
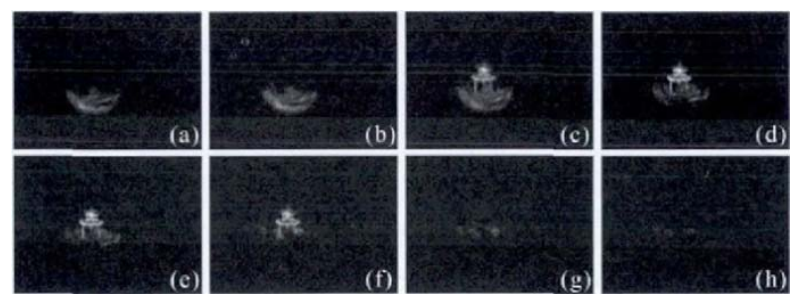

Fig. 6 Active laser imaging experiment images

\section{Analysis of Experimental Results}

The measured gray-scale curve is seriously affected by noise. Curve fitting can smooth the curve and eliminate the ambiguity in the data feature matching. Fig. 7 shows the 3D reconstruction model at the distance of $1.2 \mathrm{~km}$ based on the method of 13 times fitting of gray-scale curve and data feature positioning. 


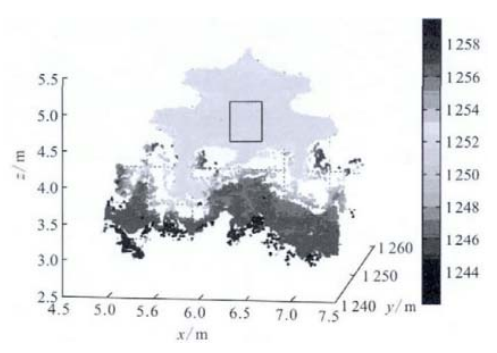

Fig. 7 Result from gray-scale curve fitting with data feature positioning

Fig. 7 shows that the arbor outline has an apparent distance of approximately $1253 \mathrm{~m}$, and the front edge is approximately $1.3 \mathrm{~m}$ long, which is basically consistent with the dimensions of the actual object. However, the concave-convex detail of the arbor is not evident in the red box.

The proposed cross-correlation method can improve the calculation accuracy of the pixel distance value and consequently the accuracy of the $3 \mathrm{D}$ reconstruction model of long-range targets to calculate the pixel range value based on the interceptive gray-scale curve. A 3D reconstruction model of the arbor at the distance of $1.2 \mathrm{~km}$ is obtained by this method as shown in Fig. 8 .

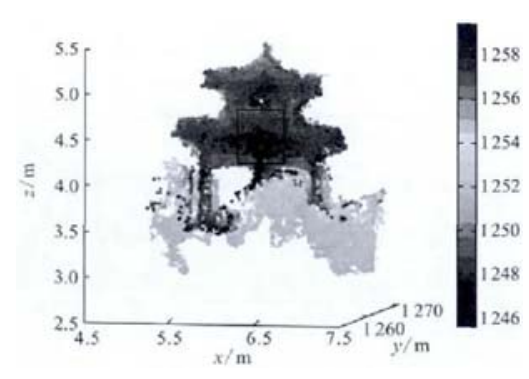

(a) Front view of the arbor

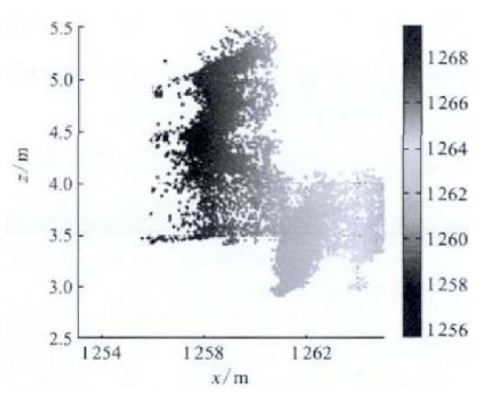

(b) Image of arbor rotates $90^{\circ}$ clockwise

Fig. 8 Result achieved by the method of the interceptive gray-scale curve with cross-correlation

Fig. 8(a) shows that the 3D reconstruction of the arbor is greatly improved compared with that shown in Fig. 7, and the concave-convex detail is evident in the red box as shown in Fig. 7. The distance is approximately $1257 \mathrm{~m}$, and the length of the front edge is approximately $1.3 \mathrm{~m}$, which is basically consistent with the dimensions of the actual object. Fig. 8(b) is the image of the arbor after rotating $90^{\circ}$ clockwise, which shows that the longitudinal distance of half of the arbor is approximately $1.9 \mathrm{~m}$ to $2.0 \mathrm{~m}$. The difference between the distance values of different pixels is calculated accurately. Although the surface noise of the arbor is relatively low, the outline of the upper and lower houses is clear. Therefore, Fig. 8(a) provides detailed information in the front of the arbor.

\section{Conclusion}

In this paper, the interceptive gray-scale curve and the cross-correlation method are used to calculate the range values of a long-range target at each pixel. The optical system parameters are used to calculate the 3D coordinates of each pixel to realize the 3D reconstruction of the long-range target. Correlated accuracy is improved by using the interceptive gray-scale curve where the zero-value point is rejected. Compared with many fitting calculations for eliminating ambiguity in the data feature positioning, this method can improve the speed of reconstruction while reducing the amount of calculation. Few reports were found on the $3 \mathrm{D}$ reconstruction of long-range targets based on rangegated laser active imaging. The present experimental results show that the proposed method can achieve high accuracy of concave-convex detail of the target surface to achieve the high-precision 3D reconstruction of long-range targets. However, due to the large amount of cross-correlation calculations required in this method, the reconstruction speed is slow, and the reconstruction model is seriously affected by noise. Thus, future works should focus on reducing the amount of cross- 
correlation calculation and de-noising 3D images to improve the speed and accuracy of the 3D reconstruction of long-range targets.

\section{References}

[1]. Tang Zhanhong, Yu Shicai. Method of three -dimensionalreconstruction based on surface rendering and its improving research [J]. Computer Engineering and Design, 2009, 30(9):22252228.

[2]. Jens Busck, Henning Herselberg . High accuracy 3 - D laser radar[C]//SPIE, 2004, 5412: 257263.

[3]. Pierre Andersson. Long -range three-dimensional imaging using range-gated laser radar images [J]. Optical Engineering ,2006, 45(3): 1-10.

[4]. Wu Fan, Zhang Yong, He Jiang, et al. Improved range-gating 3D imaging laser radar [J]. Infrared and Laser Engineering , 2011, 40(12): 2388-2392.

[5]. Bai Lianfa, Zhang Yi, Chen Qian, et al. Some questions in the realization of range gated imaging [J]. Infrared and Laser Engineering , 2009, 38(1): 57-61.

[6]. Wang Shouzeng, Sun Feng, Zhang Xin. Development of laser illuminating range -gated imaging technique [J]. Infrared and Laser Engineering, 2008, 37: 57-61.

[7]. Martin Laurenzis, Frank Christnacher, David Monnin. Long-range three-dimensional active imaging with supperresolution depth mapping [ J ] . Optics Letters , 2007 , 32 ( 21 ) : 3146 -3148.

[8]. Jens Busck, Henning Herselberg. Gated viewing and high-accuracy three-dimensional laser radar [J]. Appl Opt, 2003,43: 4705-4710.

[9]. Xiuda Z, Huimin Y, Yanbing J. Pulse-shape-free method for long-range three-dimensional active imaging with high linear accuracy[J]. Opt Let, 2008, 33: 1219. 